\title{
Confianza en decisiones sobre productos de conveniencia y de comparación ${ }^{1}$
}

DOI: http://dx.doi.org/10.17981/econcuc.37.1.2016.04

\section{José Roberto Concha Velásquez ${ }^{2}$}

\section{Resumen}

El propósito del presente estudio es analizar como interactúan los constructos: confianza en la marca, lealtad en la marca y valor percibido, en la decisión de compra de productos de conveniencia y productos de comparación. Los constructos en este estudio fueron evaluados utilizando escalas de medidas desarrolladas en anteriores investigaciones. Se demuestra que dichos elementos actúan de igual manera cuando se trata de productos de conveniencia y de comparación. Los resultados muestran una fuerte relación entre las variables, explicando como la confianza de marca y el valor influyen positivamente sobre la lealtad en la compra de los consumidores al momento de tomar su decisión en el caso de productos de conveniencia y de comparación.

Palabras clave: confianza; lealtad, valor; productos de conveniencia; productos de comparación.

Recibido: 15.9.2015 Devuelto para revisión: 20.10.2015 Aceptado: 26.11.2016

\begin{abstract}
${ }^{1}$ Artículo científico derivado de la investigación "Desarrollo de confianza en decisiones sobre productos de conveniencia y de comparación”, financiada por la Universidad ICESI, Cali, Colombia.

${ }^{2}$ PhD Business. Master of Management. Tulane University-New Orleans, Estados Unidos. Magister en Ingeniería Industrial y de Sistemas. Ingeniero químico, Universidad del Valle- Cali. Colombia. Jefe del Departamento de Mercadeo y Negocios Internacionales, adscrito al grupo de investigación en Mercadeo y Competitividad de las Organizaciones. Universidad ICESI, Cali, Colombia.jrconcha@icesi.edu.co
\end{abstract}

- The author; licensee Universidad de la Costa - CUC 


\title{
Trust on Decisions Regarding Convenience and Shopping Products
}

\begin{abstract}
The purpose of this research study is to present a discussion of the interaction among the following constructs: brand trust, brand loyalty, and perceived value in making decisions regarding the purchase of convenience products (shampoo) and shopping products (lap top computers). The constructs in review in this study were evaluated using rating scales developed in previous research studies. It is reveals that these factors work the same way when it comes to convenience and shopping products. The findings show that there is a strong relationship between variables and explain how brand trust and value have a positive influence on consumers' purchase loyalty when making a decision about convenience and shopping products.
\end{abstract}

Keywords: trust; loyalty; value; shopping products; convenience products.

\section{Introducción}

En la actualidad, las organizaciones se encuentran en la búsqueda de nuevas formas de incrementar la confianza en la marca, la intención de compra y la lealtad de marca, de tal manera que la frecuencia de compra de sus clientes sea recurrente y se creen relaciones redituables y a largo plazo con ellos (Bloemer y Kasper, 1995).

La lealtad que se genera en el cliente con una marca es primordial para la subsistencia de una organización (Oliver, 1999), ya que la marca es uno de los principales activos que logra generar ingresos con el solo hecho de impactar en un segmento o grupo de personas específico (Ranaweera y Prabhu, 2003).
Es importante aclarar que la marca debe satisfacer las necesidades y requerimientos de los consumidores para así generar un vínculo emocional con la misma (Ballester-Delgado y Aleman-Munuera, 2001). Sin embargo, los vínculos que se generen desde los consumidores para con la marca pueden ser de tipo positivo cuando los consumidores generan recompra y escogen la marca por encima de otras, o negativo cuando sus necesidades no se ven satisfechas y recurren a otras marcas (Cunningham, 1956).

La lealtad de marca es uno de los factores más importantes en el mundo competitivo empresarial global (Sirdeshmukh, Singh y Sabol, 2002); las empresas buscan que sus marcas se encuentren en el top of mind $y$ 
top of heart de los consumidores, por esto, generan diferentes estrategias para captar la lealtad de los clientes y que así escojan su marca como la número uno (Chaudhuri y Holbrook, 2001).

Esta investigación se desarrolla con fin de conocer cómo se interrelacionan el desarrollo de confianza de los clientes, la lealtad de marca que esos clientes tienen por un producto y el valor percibido por el cliente, en el caso de productos de conveniencia (champú) y productos de comparación (computadores).

\section{Definiciones}

Con el propósito de unificar conceptos desarrollados a lo largo de esta investigación, se definirán los términos claves aquí utilizados:

Marca. Chaudhuri y Holbrook (2001) consideran que "ya sea que se trate de un nombre, una marca comercial, un logotipo u otro símbolo, una marca es en esencia la promesa de una parte vendedora de proporcionar, de forma consistente a los compradores, un conjunto específico de características, beneficios y servicios". Las compañías se encuentran en una constante búsqueda y desarrollo de marcas que tengan como objetivo la satisfacción de las necesidades de los clientes, logrando así que estos creen relaciones y asociaciones de carácter positivo con ella (Kim y Lee, 2008).
La marca es uno de los factores más importantes en el mundo competitivo empresarial, ya que ayuda a los consumidores, en el momento de la compra, a la selección del producto; sin embargo, se deben tener en cuenta otras características como el precio, el empaque, la disponibilidad del producto, entre otras (Cronin, Brady y Hult, 2000).

Por último, es posible afirmar que una marca es importante para una organización ya que es una herramienta que ayuda a que los consumidores adquieran el producto y continúen comprando a través del tiempo (Lam, Shankar, Erramilli y Murthy, 2004).

Valor percibido. Se define como el resultado de la comparación por parte del consumidor de los beneficios percibidos y los sacrificios realizados (Zeithaml, 1988 ) (McDougall y Levesque, 2000). Es posible decir que el valor percibido es un proceso subjetivo que varía de cliente a cliente, ya que cada uno de ellos se encarga de analizar y equilibrar los beneficios que puede recibir al adquirir una marca u otra, así mismo, las opciones que tiene a su disposición gracias a la amplitud del mercado (Kim y Lee, 2008). Por otra parte, es sabido que el valor percibido por los consumidores tiene un reflejo directo sobre la actitud del cliente para con la marca o producto, ya que es el encargado de dar un juicio y tomar decisiones sobre si adquiere el producto o no, teniendo en cuenta el valor percibido (Cronin et al., 2000). 
Lealtad de marca. "La conducta en la cual algunas personas tienden a adquirir siempre la misma marca cuando van de compras y ese hábito responde a un acto de conciencia en la elección del producto adquirido" (Solomon, Bamossy, Askegaard y Hogg, 2006). La lealtad de marca es uno de los factores más importantes que influyen en la intención de compra de los consumidores, ya que al lograr que los clientes de una marca se enganchen emocional y racionalmente, la vulnerabilidad de la base de clientes a las acciones de la competencia se reduce (Gounaris, Dimitriadis y Stathakopoulos, 2005 ), así por ejemplo, si la competencia realizara el lanzamiento de un nuevo producto, los clientes fieles o leales a la marca otorgaran a la compañía el tiempo necesario para igualar o superar el producto de la competencia.

"El costo de atraer nuevos consumidores se ha encontrado seis veces mayor que el costo de retener a los actuales" (Rosenberg y Czepiel, 1983). Al generar propuestas de valor atractivas para los clientes, lo que se busca es generar relaciones a largo plazo con los mismos, para, de esta manera, construir una base sólida de consumidores. Por otra parte, se sabe que la construcción de nuevas relaciones genera mayores costos; es por esto que las compañías generan grandes campañas en medios, buscan estar al pendiente de las necesidades de los clientes ya existentes y generar valor en sus marcas o productos que sea fácilmente percibido por los consumidores para crear en ellos el sentimiento de lealtad y, así, generar relaciones redituables con los mismos. Además, es posible decir que al hacer que los consumidores sean leales hacia una marca específica se logra crear una importante barrera ante los competidores, obteniendo como resultado una gran participación en el mercado.

"La lealtad de marca crea clientes satisfechos, lo cual es muy importante, ya que un cliente no satisfecho comenta en promedio a dieciocho personas su mala experiencia" (Bigne y Blesa, 2003). Las empresas saben que la lealtad de un cliente hacia una marca o producto no solo está en crear buenos productos, está en el hecho de que los consumidores sientan y consientan que sus necesidades son más que satisfechas. De este modo, la empresa adquiere un lugar importante en la mente del consumidor, logrando que éste, sin quererlo, divulgue su experiencia $y$, por ende, incitando a otros a probar y engancharse con la marca o producto (Bansal, Irving y Taylor, 2004 ).

Intención de compra. Al hablar de la intención de compra de un cliente, se refiere específicamente a la declaración de la preferencia por una marca o producto que está por encima del resto de las opciones competidoras. Es un dato declarativo por parte 
del grupo objetivo y, por lo tanto, no siempre corresponde con las ventas reales, que reflejan el comportamiento y no la intención (Olsen, 2002). Es posible decir que la intención de compra es únicamente una actitud mental que puede o no suceder. Se puede entender como una situación probable; es por esto que las compañías no deben confiarse totalmente de la lealtad de sus clientes.

Para lograr que la intención de compra se convierta en una realidad y suceda, las empresas deben desplegar todo su conocimiento sobre mercados potenciales, generación de valor, innovación en nuevos productos, precios competitivos pero rentables al tiempo, necesidades del cliente etc., que le permita a los clientes tener dentro de sus opciones la marca o producto de la compañía; ahora bien, al lograr ubicarse en las opciones posibles del consumidor será mucho más probable ser seleccionados por el mismo (Cadogan y Foster, 2000).

\section{Modelo de Investigacion}

El autor propone un modelo de investigación (figura 1) que relaciona la confianza en la marca, el valor percibido y la lealtad en la marca. Este modelo ya ha sido estudiado previamente por diversos autores, pero lo novedoso en este estudio es que se busca evaluar si el modelo es aplicable por igual a productos de conveniencia y a productos de comparación. Para tal efecto, se desarrollaron dos investigaciones diferentes: para la categoría de champú en la línea de productos de conveniencia y para la categoría de computadores en la línea de productos de comparación.

Este modelo supone dos hipótesis básicas:

H1: la confianza en la marca tiene un efecto positivo en la lealtad de la marca.

H2: el valor percibido por el consumidor tiene un efecto positivo en la lealtad en la marca del bien adquirido.

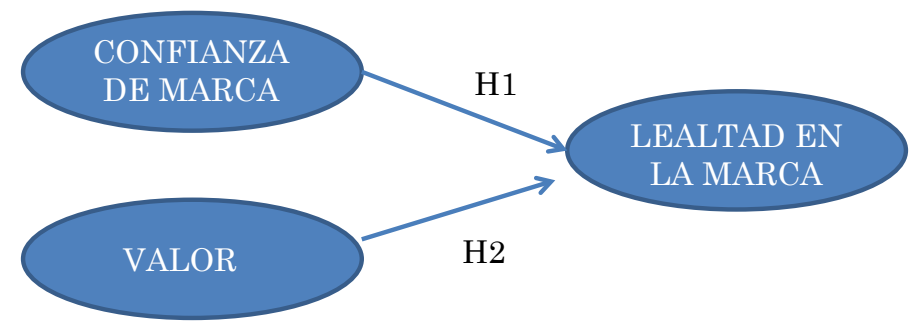

Figura 1. Modelo de Investigación Fuente: Abraheem, (2012) 


\section{Metodología}

Mediciones. Todos los constructos fueron medidos utilizando escalas adoptadas de estudios anteriores:

Para la evaluación de confianza se recurrió a las escalas probadas por Morgan y Hunt (1994), Ballester-Delgado y Aleman-Munuera (2001) y Chaudhuri y Holbrook (2001), usando una escala de likert de 5 puntos, donde uno (1) equivale a totalmente en desacuerdo y cinco (5) equivale a totalmente de acuerdo. Para el constructo valor percibido se utilizaron también escalas utilizadas en estudios previos (Mathwick, Malhotra y Rigdon, 2001; Petrick, 2002; Sweenwy y Soutar, 2001). Y finalmente, para evaluar la lealtad de marca, se analizaron escalas previas utilizadas en estudios anteriores también (Chaudhuri y Holbrook, 2001; Fullerton, 2005; Hess y Story, 2005).

Muestra. La muestra escogida para esta investigación son hombres y mujeres con edades entre los 17 y 45 años de edad, quienes fueron escogidos aleatoriamente y usan diferentes marcas de champú y computadores. Las marcas de champú evaluadas fueron Sedal, Pantene, Head \& Shoulders, Herbal Essences,
Konzil y Kerastase; y las marcas evaluadas para computadores fueron Acer, Compaq, Dell, Hewlett-Packard, Asus, Apple, Lenovo, Toshiba, Sony y Samsung.

El propósito de realizar esta investigación con estas dos categorías de producto, champú y computadores, está relacionada con el hecho de que a nivel de productos de conveniencia, el champú es un artículo de fácil compra y/o adquisición que genera niveles de lealtad o confianza en su uso, tanto en hombres como en mujeres. Por otra parte, se tomó la categoría de computadores para investigarla, ya que a la hora de adquirir un computador los consumidores tienden de igual manera a analizar más los atributos o beneficios que ofrece una marca u otra, pues su adquisición implica un importante desembolso de dinero y lo que el consumidor busca es una serie de características importantes como capacidad, velocidad, durabilidad, rendimiento de la máquina, etc.

La muestra está compuesta por cuatrocientas (400) personas con un porcentaje de participación masculina del $33,50 \%$ y un $66,50 \%$ de participación femenina. La Tabla 1 muestra las características de esa muestra. 
Tabla 1.

Perfil de la Muestra

\begin{tabular}{cccc}
\hline & & Frecuencia & $\%$ \\
\hline \multirow{2}{*}{ Edad } & Menos de 20 años & 114 & 28,5 \\
& $20-25$ años & 230 & 27,5 \\
& 25 a 30 años & 12 & 3 \\
& Más de 30 años & 44 & 11 \\
\hline \multirow{2}{*}{ Género } & Total & $\mathbf{4 0 0}$ & $\mathbf{1 0 0}$ \\
& Femenino & 266 & 66,5 \\
& Masculino & 134 & 33,5 \\
\hline
\end{tabular}

Fuente: elaboración propia.

\section{Recopilacion de Datos}

El instrumento utilizado para la recopilación de los datos fue una encuesta auto-administrada, la cual evaluaba dos categorías de productos: champú y computadores. Ésta iniciaba con preguntas de control como edad y género, seguida de una pequeña introducción que sugiere la confidencialidad de la información; de igual manera, se nombraron diferentes marcas, tanto de champú como de computadores, para conocer la recordación de éstas en los encuestados.

\section{Resultados y Análisis}

Para la categoría de champú hay once (11) ítems que miden el constructo lealtad, seis (6) para confianza de marca y cuatro (4) para el valor percibido; lo anterior se puede observar en la Tabla 2. Los tres factores mencionados anteriormente presentan una varianza de $50.025 \%$, $6.646 \%$ y $5.870 \%$, respectivamente. Según investigaciones anteriores un Cronbach's Alpha superior a 0.70 es considerado generalmente confiable (Nunnally y Bernstein, 1994). Para este estudio el estadístico Cronbach's Alpha fue de $0.930,0.851$ y 0.595 , respectivamente. $\mathrm{El}$ factor loading tiene un rango de entre 0.231 y 0.843 . A continuación se muestra en la tabla lo mencionado anteriormente. 
Tabla 2.

Análisis Factorial del estudio de las variables para Champú.

\begin{tabular}{|c|c|c|c|c|c|}
\hline & \multirow{2}{*}{$\begin{array}{l}\text { Factor } \\
\text { loading }\end{array}$} & \multirow{2}{*}{$\begin{array}{l}\text { Eigen } \\
\text { value }\end{array}$} & \multirow{2}{*}{$\begin{array}{c}\% \\
\text { Variance }\end{array}$} & \multirow{2}{*}{ Reliability } & \multirow{2}{*}{ Construct } \\
\hline & & & & & \\
\hline Considerare comprar esta marca como mi primera opción & 0.843 & \multirow{11}{*}{10.505} & \multirow{11}{*}{50.025} & \multirow{11}{*}{0.930} & \multirow{11}{*}{ Lealtad } \\
\hline La marca de mi producto es conveniente & 0.818 & & & & \\
\hline Creo en la marca & 0.800 & & & & \\
\hline Confio en la marca & 0.799 & & & & \\
\hline Intentaré recomendar la marca a otras personas & 0.767 & & & & \\
\hline El valor agregado que recibo es justo & 0.758 & & & & \\
\hline Intentaré comprra la marca en un future cercano & 0.754 & & & & \\
\hline $\begin{array}{l}\text { La próxima vez que necesite el product, compraré la } \\
\text { misma marca }\end{array}$ & 0.751 & & & & \\
\hline Seguiré siendo un consumidor leal a esta marca & 0.748 & & & & \\
\hline Podría decir cosas positivas de la marca & 0.496 & & & & \\
\hline Intentaré pagar un precio premium & 0.327 & & & & \\
\hline $\begin{array}{l}\text { Consideraré comprar esta marca como primera opción el } \\
\text { próximo año }\end{array}$ & 0.711 & \multirow{6}{*}{1.396} & \multirow{6}{*}{6.646} & \multirow{6}{*}{0.851} & \multirow{6}{*}{$\begin{array}{l}\text { Confianza } \\
\text { de Marca }\end{array}$} \\
\hline La marca es honesta & 0.709 & & & & \\
\hline $\begin{array}{l}\text { La marca me ofrece un valor equitativo al precio que } \\
\text { pago }\end{array}$ & 0.330 & & & & \\
\hline La marca es segura & 0.312 & & & & \\
\hline La marca llena mis expectativas & 0.307 & & & & \\
\hline Podría recomendar la marca & 0.291 & & & & \\
\hline $\begin{array}{l}\text { Sólo compraría esta marca de nuevo si continua } \\
\text { económica. }\end{array}$ & 0.707 & \multirow{4}{*}{1.233} & \multirow{4}{*}{5.870} & \multirow{4}{*}{0.595} & \multirow{4}{*}{$\begin{array}{l}\text { Valor } \\
\text { Percibido }\end{array}$} \\
\hline La marca tiene un precio razonable & 0.683 & & & & \\
\hline La marca me ofrece un buen product en relación al precio & 0.268 & & & & \\
\hline Intentaré comprar otros productos de la marca & 0.231 & & & & \\
\hline
\end{tabular}

Fuente: elaboración propia. 
Tabla 3.

Análisis Factorial del estudio de las variables para Computadores

\begin{tabular}{|c|c|c|c|c|c|}
\hline & \multirow{2}{*}{$\begin{array}{l}\text { Factor } \\
\text { loading }\end{array}$} & \multirow{2}{*}{$\begin{array}{l}\text { Eigen } \\
\text { value }\end{array}$} & \multirow{2}{*}{$\frac{\%}{\text { Variance }}$} & \multirow{2}{*}{ Reliability } & \multirow{2}{*}{ Construct } \\
\hline & & & & & \\
\hline Creo en la marca & 0.878 & \multirow{11}{*}{12.038} & \multirow{11}{*}{57.323} & \multirow{11}{*}{0.960} & \multirow{11}{*}{ Lealtad } \\
\hline Consideraré comprar esta marca como mi primera opción & 0.863 & & & & \\
\hline Podría decir cosas positivas de la marca & 0.850 & & & & \\
\hline Intentaré comprar la marca en un futuro cercano & 0.846 & & & & \\
\hline Seguiré siendo un consumidor leal a esta marca & 0,845 & & & & \\
\hline $\begin{array}{l}\text { La próxima vez que necesite el producto, compraré la } \\
\text { misma marca }\end{array}$ & 0.838 & & & & \\
\hline Intentaré recomendar la marca a otras personas & 0.834 & & & & \\
\hline La marca de mi producto es conveniente & 0.832 & & & & \\
\hline Creo en la marca & 0.830 & & & & \\
\hline Intentaré pagar un precio premium & 0.592 & & & & \\
\hline El valor agregado que recibo es justo & 0.539 & & & & \\
\hline Podría recomendar la marca & 0.885 & \multirow{6}{*}{1.982} & \multirow{6}{*}{9.438} & \multirow{6}{*}{0.859} & \multirow{6}{*}{ Confianza } \\
\hline $\begin{array}{l}\text { La marca me ofrece un buen product en relación al } \\
\text { precio. }\end{array}$ & 0.819 & & & & \\
\hline La marca es segura & 0.810 & & & & \\
\hline La marca llena mis expectativas & 0.613 & & & & \\
\hline $\begin{array}{l}\text { Consideraré comprar esta marca como mi primera opción } \\
\text { el próximo año. }\end{array}$ & 0.607 & & & & \\
\hline La marca es honesta & 0.550 & & & & \\
\hline $\begin{array}{l}\text { Sólo compraré esta marca de nuevo si continua } \\
\text { económica }\end{array}$ & 0.570 & \multirow{4}{*}{1.091} & \multirow{4}{*}{5.196} & \multirow{4}{*}{0.648} & \multirow{4}{*}{$\begin{array}{l}\text { Valor } \\
\text { Percibido }\end{array}$} \\
\hline La marca tiene un precio razonable & 0.330 & & & & \\
\hline $\begin{array}{l}\text { La marca me ofrece un valor equitativo al precio que } \\
\text { pago }\end{array}$ & 0.281 & & & & \\
\hline Intentaré comprar otros productos de la marca & 0.187 & & & & \\
\hline
\end{tabular}

Fuente: elaboración propia. 
Por otra parte, para la categoría de computadores hay también once (11) ítems que miden el constructo lealtad, seis (6) para confianza y cuatro (4) para precio; lo anterior se puede observaren la Tabla 3. Los tres factores mencionados anteriormente presentan una varianza de $57.323 \%$, 9.438\% y $5.196 \%$, respectivamente. Según investigaciones anteriores un Cronbach's Alpha superior a 0.70 es considerado generalmente confiable (Nunnally y Bernstein, 1994). Para este estudio el estadístico Cronbach's Alpha fue de $0.960,0.859$ y 0.648 , respectivamente. El factor loading tiene un rango de entre 0.187 y 0.878 . A continuación se muestra en una tabla lo mencionado anteriormente.
Análisis de correlación: relaciones entre las variables. Según las medias y desviación estándar calculada para cada constructo y categoría se creó una matriz de correlación. Las medias, desviaciones estándar y la correlación entre todas las escalas usadas en el análisis se muestran en las tablas 4 y 5 . Las correlaciones positivas están en el nivel $\mathrm{P}<0.01$. La matriz de correlación fue elaborada usando las variables de la encuesta con el fin de mostrar la fortaleza de la relación entre las mismas. Según Kline (1998), la matriz de correlación es definida como "un conjunto de coeficientes de correlación entre un número de variables".

Tabla 4:

Resumen de las Medias, Variaciones estándar y Correlaciones de Lealtad, Confianza y Valor Percibido de la categoría Computadores

\begin{tabular}{|c|c|c|c|c|c|}
\hline Variables & Media & Desviación Estándar & Lealtad & Confianza & Valor Percibido \\
\hline Lealtad & 4,0545 & 0,81973 & 1 & $0,669 * *$ & $0,791 * *$ \\
\hline Confianza & 4,056 & 0,73455 & & 1 & $0,624^{* *}$ \\
\hline Valor Percibido & 3,875 & 0,65384 & & & 1 \\
\hline
\end{tabular}

Fuente: Elaboración propia.

Tabla 5 .

Resumen de las Medias, Variaciones estándar y Correlaciones de Lealtad, Confianza y Valor Percibido de la categoría Champú

\begin{tabular}{|c|c|c|c|c|c|}
\hline Variables & Media & Desviación Estándar & Lealtad & Confianza & Valor Percibido \\
\hline Lealtad & 3,8473 & 0,7276 & 1 & $0,847 * *$ & $0,709 * *$ \\
\hline Confianza & 3,9867 & 0,6904 & & 1 & $0,683^{* *}$ \\
\hline Valor Percibido & 3,7938 & 0,65039 & & & 1 \\
\hline
\end{tabular}

Fuente: elaboración propia. 
Como se muestra en la Tabla 4, la matriz de correlación indica que la confianza y el precio fueron positiva $\mathrm{y}$ moderadamente correlacionadas con la lealtad. El coeficiente más alto de correlación en este estudio es 0,791, el cual está por debajo de 0,90, lo que lo excluye del problema de colinealidad. Así, el problema de multicolinealidad no está presente en este estudio (Hair, Anderson, Tatham y Black, 1998). Estas correlaciones también son una prueba de validez y fiabilidad de las escalas de medición usadas en este estudio.

Por otro lado, como se muestra en la Tabla 5, la matriz de correlación indica que la confianza de marca y el precio fueron positiva y moderadamente correlacionadas con la intención de compra. El coeficiente más alto de correlación en este estudio es 0,847 , el cual está por debajo de 0,90 , lo que lo excluye del problema de colinealidad. Así, el problema de multicolinealidad no está presente en este estudio (Hair et al., 1998). Estas correlaciones también son una prueba de validez y fiabilidad de las escalas de medición usadas en este estudio.

Análisis de regresión. Se aplicó el análisis de la regresión vía SPSS. Para la categoría de computadores, los constructos confianza y valor percibido, representan las variables independientes, y el constructo lealtad de marca representa la variable dependiente. El modelo de regresión fue estadísticamente significante $(\mathrm{F}=$ 205.106 $\left.\mathrm{R}^{\wedge} 2=0.822 \mathrm{P}=0.000\right)$. El análisis de regresión indicó que las variables confianza y precio tienen un efecto positivo significante en la variable lealtad $(p<0.05$; $\beta=0.288)$ y $(p<0.05 ; \beta=0.611)$, respectivamente.

Tabla 6.

Resumen de la Regresión realizada para medir el impacto de la Confianza y el Valor Percibido sobre la Lealtad en la categoría Computadores $(N=400)$

\begin{tabular}{|c|c|c|c|c|c|c|c|c|}
\hline & \multirow{2}{*}{ Modelo } & \multicolumn{2}{|c|}{$\begin{array}{l}\text { Coeficientes no } \\
\text { estandarizados }\end{array}$} & \multirow{2}{*}{$\begin{array}{c}\begin{array}{c}\text { Coeficientes } \\
\text { Tipificados }\end{array} \\
\text { Beta }\end{array}$} & \multirow{2}{*}{$\mathbf{t}$} & \multirow{2}{*}{$\begin{array}{l}\text { Sig. } \\
\text { Tolerancia }\end{array}$} & \multicolumn{2}{|c|}{$\begin{array}{c}\text { Estadísticos de } \\
\text { Colinealidad }\end{array}$} \\
\hline & & B & $\begin{array}{l}\text { Error } \\
\text { Típico }\end{array}$ & & & & FIV & \\
\hline \multirow{3}{*}{1} & (Constante) & $-0,216$ & 0,214 & & $-1,008$ & 0,315 & & \\
\hline & Confianza & 0,321 & 0,058 & 0,288 & 5,536 & 0 & 0,611 & 1,638 \\
\hline & Valor Percibido & 0,766 & 0,065 & 0,611 & 11,769 & 0 & 0,611 & 1,638 \\
\hline
\end{tabular}

Fuente: elaboración propia. 
Así, H1 propone que la confianza en la marca tiene un efecto positivo en la lealtad de la marca. A su vez, H2 señala que el valor percibido por el consumidor tiene un efecto positivo en la lealtad en la marca del bien adquirido. De esta forma, ambas hipótesis son aceptadas y soportadas por este estudio. Esta información se puede observar en la Tabla 6.

Por otro lado, para la categoría de champú, los constructos confianza de marca y valor percibido, representan las variables independientes, y el constructo intención de compra, representa la variable dependiente. El modelo de regresión fue estadísticamente significante $(\mathrm{F}=207.103$
$\left.\mathrm{R}^{\wedge} 2=.0723 \mathrm{P}=0.000\right)$. El análisis de regresión indicó que las variables confianza de marca y valor percibido por el cliente tienen un efecto positivo significante en la variable intención de compra $(p<0.05$; $B=0.288)$ y $(p<0.05 ; \beta=0.611)$, respectivamente.

Así, H1 propone que la confianza en la marca tiene un efecto positivo en la lealtad de la marca. A su vez, H2 dice que el valor percibido por el consumidor tiene un efecto positivo en la lealtad en la marca del bien adquirido. Entonces, ambas hipótesis son aceptadas y soportadas por este estudio. Esta información se puede observar en la Tabla 7.

Tabla 7.

Resumen de la Regresión realizada para medir el impacto de la Confianza y el Valor Percibido sobre la Lealtad en la categoría Champu $(N=400)$

\begin{tabular}{|c|c|c|c|c|c|c|c|c|}
\hline & \multirow{2}{*}{ Modelo } & \multicolumn{2}{|c|}{$\begin{array}{l}\text { Coeficientes no } \\
\text { Estandarizados }\end{array}$} & \multirow{2}{*}{$\begin{array}{c}\begin{array}{c}\text { Coeficientes } \\
\text { Tipificados }\end{array} \\
\text { Beta }\end{array}$} & \multirow{2}{*}{$\mathbf{t}$} & \multirow{2}{*}{$\begin{array}{l}\text { Sig. } \\
\text { Tolerancia }\end{array}$} & \multicolumn{2}{|c|}{$\begin{array}{l}\text { Estadísticos de } \\
\text { Colinealidad }\end{array}$} \\
\hline & & B & $\begin{array}{l}\text { Error } \\
\text { Típico }\end{array}$ & & & & FIV & \\
\hline \multirow{3}{*}{1} & (Constante) & $-0,047$ & 0,166 & & $-0,285$ & 0,776 & & \\
\hline & Confianza & 0,717 & 0,051 & 0,68 & 13,936 & 0 & 0,534 & 1,872 \\
\hline & Valor Percibido & 0,273 & 0,055 & 0,244 & 5,001 & 0 & 0,534 & 1,872 \\
\hline
\end{tabular}

Fuente: elaboración propia. 


\section{Conclusiones}

El propósito de este estudio es presentar el rol de la confianza de marca y el valor percibido como herramientas de marketing para asegurar la lealtad de marca del cliente en productos de conveniencia y de comparación.

Con la ayuda de escalas de medición desarrolladas en estudios previos, se elaboró una herramienta que posee en total 21 preguntas, con once ítems que miden la lealtad de los consumidores al momento de comprar, los siguientes seis miden la confianza y los cuatro ítems restantes hacen referencia al valor percibido. Con este instrumento se evaluó la confiabilidad y la validez de los constructos mencionados anteriormente. Los resultados mostraron que la relación entre dichas variables es válida y segura, tanto en productos de conveniencia como en productos de comparación.

Los anteriores resultados muestran, desde el punto gerencial, que las empresas deben generar estrategias de mercadeo utilizando los factores de valor percibido y desarrollo de confianza en la marca para lograr que el consumidor desarrolle lealtad en el producto o servicio $\mathrm{y}$, de esta manera, puedan aumentar las ventas.

\section{Referencias}

Abraheem, A. (2012). The Effect of Brand Trust and Perceived Value in Building Brand Loyalty. International Research. Journal of Finance and Economics, 85. 111-126.

Ballester-Delgado, E. y Aleman-Munuera, J. (2001). Brand Trust in the context of consumer loyalty. European Journal of Marketing, 35(11/12).1238-1258.

Bansal, H., Irving, P., y Taylor, S. (2004). A Three-Component Model of Customer Commitment to Service Providers. Journal of the Academy of Marketing Science, 32(3). 234-250.

Bigne, E. y Blesa, A. (2003). Market orientation, trust and satisfaction in dyadic relationships: A manufacturer retailer analysis. International Journal of Retail \& Distribution Management, 31(11). 574-590.

Bloemer, J. y Kasper, H. (1995). The Complex Relationship between Consumer Satisfaction and Brand Loyalty. Journal of Economic Psychology, 16(2). 311-329. 
Cadogan, J. y Foster, B. (2000). Relationship Selling and Customer Loyalty: An Empirical Investigation. Marketing Intelligence and Planning, 18(4).185-199.

Chaudhuri, A. y Holbrook, M. (2001). The chain of effects from brand trust and brand affect to brand performance: The role of brand loyalty. Journal of Marketing, 65(2). 81-93.

Cronin, J., Brady, M., y Hult, G. (2000). Assessing the effects of quality, value, and customer satisfaction on consumer behavioral intentions in service environments. Journal of Retailing, 76(2). 193-218.

Cunningham, R. (1956). Brand Loyalty, what, where, how much? Harvard Business Review, 34(1).116128.

Fullerton, G. (2005). The Impact of Brand Commitment on Loyalty to Retail Service Brands. Canadian Journal of Administratiand Sciences, 22(2). 97-110.
Gounaris, S., Dimitriadis, S. y Stathakopoulos, V. (2005). An examination of the effects of service quality and satisfaction on customers' behavioral intentions in eshopping. Journal of services marketing, 24 (2). 142-156.

Hair, J., Anderson, R., Tatham, R. y Black, W. (1998). Multivariate Data Analysis (5th Edition). Upper Saddle River: Prentice-Hall International.

Hess, J. y Story, J. (2005). Trust-based commitment: Multidimensional consumer-brand relationships. Journal of Consumer Marketing, 22(6). 313-322.

Kim, J., y Lee, H. (2008). Consumer product search and purchase behavior using various retail channels: The role of perceived usefulness. International Journal of Consumer Studies, 32(6). 619-627.

Kline, R. (1998). Principles and Practice of Structural Equation Modeling. New York: The Guilford Press. 
Lam, S., Shankar, V., Erramilli, M., y Murthy, B. (2004). Customer value, satisfaction, loyalty, and switching costs: An illustration from a business to business service context. Journal of the Academy of Marketing Science, 32(3). 293-311.

Mathwick, C., N. Malhotra, y E. Rigdon (2001). Experiential Value: Conceptualization, Measurement and Application in the Catalog and Internet Shopping Environment. Journal of Retailing, 77(1). 39-56.

McDougall, G. y Levesque, T. (2000). Customer satisfaction with services: putting perceived value into the equation. Journal of Services Marketing, 14(5). 392-410.

Morgan, R. y Hunt, S. (1994). The commitment-trust theory of relationship marketing. Journal of Marketing, 58(7). 20-38.

Nunnally, J. y Bernstein, I. (1994). Psychometric Theory (3 ed.). New York: McGraw-Hill.

Oliver, R. (1999). Whence consumer loyalty? Journal of Marketing, 63(1). 33-44.
Olsen, S. (2002). Comparative Evaluation and the relationship between quality, satisfaction, and repurchase loyalty. Journal of the Academy of Marketing Science, 30(3). 240249.

Petrick, J. (2002). Development of a Multi-Dimensional Scale for Measuring the Perceived Value of a Service. Journal of Leisure Research, 34(2). 119-134.

Ranaweera, C. y Prabhu, J. (2003). The influence of satisfaction, trust and switching barriers on customer retention in a continuous purchasing setting. International. Journal of Service Industry Management, 14(4). 374-395.

Rosenberg. L. y Czepiel, J. (1983). A Marketing Approach to Customer Retention. Journal of Consumer Marketing, 1(2). 45-51.

Sirdeshmukh, D., Singh, J. y Sabol, B. (2002). Consumer trust, value, and loyalty in relational exchanges. Journal of Marketing, 66(1). 15-37. 
Solomon, M., Bamossy, G., Askegaard, S. y Hogg, M. (2006). Consumer Behaviour: A European Perspective 3rd ed. Londres: Pearson Education.
Zeithaml, V. (1988). Consumer perceptions of price, quality, and value: A means-end model and synthesis of evidence, Journal of Marketing, 52(3). 2-22. 